Original Paper http://ajol.info/index.php/ijbcs http://indexmedicus.afro.who.int

\title{
Effets comparés de rations à base des variétés de maïs « ESPOIR » et de maïs «SR21 » Sur la productivité du poulet de chair de souche cobb-500
}

\author{
O.C. HIEN ${ }^{1 *}$, I. SALISSOU ${ }^{2}$, A. OUEDRAOGO ${ }^{3}$, L. OUATTARA ${ }^{3}$, \\ B. Diarra ${ }^{3}$ et J.D. HANCOCK ${ }^{4}$

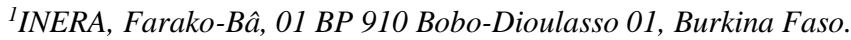 \\ ${ }^{2}$ INRAN BP 429 Niamey, Niger. \\ ${ }^{3}$ UNB, IDR 01 BP 1091, Bobo-Dioulasso 01, Burkina Faso. \\ ${ }^{4}$ Dept of Animal Science and Industry, Manhatan KS 66506-0201. \\ *Auteur correspondant; E-mail: hien_ollo@yahoo.fr
}

\section{RESUME}

Le Burkina Faso, pays à vocation agropastorale, accorde une grande importance à l'aviculture, confrontée à plusieurs difficultés notamment celle liée à l'alimentation; le maïs constitue la composante essentielle les rations alimentaires destinées à la volaille, Cette étude avait pour but d'appréhender les effets de l'incorporation de variétés de maïs plus riches en protéines que le maïs tout venant sur les performances zootechniques du poulet de chair. Pour ce faire, deux rations contenant la variété de maïs Espoir (ESPOIR) et la variété de maïs blanc (SR21) ont été comparées à une troisième qui était un aliment commercial contenant du maïs tout venant (CPAVI). 207 poussins d'un jour de poids moyen 41,8 g, de souche Cobb-500 ont été répartis de façon aléatoire en 9 lots de 23 poulets chacun. Les résultats ont montré que l'effet variété de maïs n'a pas été significatif sur la consommation alimentaire, l'IC, le GMQ et le rendement carcasse. Par contre les PVM des poulets sous traitements ESPOIR et SR21 étaient similaires mais tous deux supérieurs à celui des sujets sur CPAVI au $42^{\mathrm{e}}$ jour : $1743,8 \pm 248$ et $1721,3 \pm 250,2$ contre $1610,3 \pm 244,9 \mathrm{~g}$ respectivement. En dehors du rendement en patte, les organes étaient plus lourds avec CPAVI. Sur le plan économique, des bénéfices de 484 f CFA, 345 f CFA et 281 f CFA ont été obtenues sur les traitements SR21, ESPOIR et CPAVI respectivement. Par conséquent, les deux variétés «SR21 » et «ESPOIR » sont meilleures que le maïs tout venant dans l'alimentation des poulets de chair.

(C) 2018 International Formulae Group. All rights reserved.

Mots clés: Maïs, ESPOIR, SR21, protéines, cobb-500, Burkina Faso.

\section{Comparative effects of rations based on "ESPOIR" and "SR21" corn varieties on the productivity of the cobb-500 broiler}

\begin{abstract}
Burkina Faso, a country with an agro-pastoral vocation, attaches great importance to poultry farming, faced with several difficulties, particularly those related to food; maize is the essential component of food rations for poultry. The aim of this study was to understand the effects of incorporating more rich protein maize varieties than corn on the zootechnical performance of broiler chickens. For this purpose, two rations containing the "ESPOIR" variety of maize and the white maize variety "SR21" were compared to a third which was a
\end{abstract}


commercial feed (CPAVI) containing all coming maize. 207 one-day old chicks weighing $41.8 \mathrm{~g}$, of Cobb-500 strain were randomly distributed in 9 lots of 23 chickens each. The results showed that the variety effect of maize was not significant on food consumption, IC, GMQ and carcass yield. On the other hand, average live weight of the chickens on treatments ESPOIR and SR21 were similar but both were higher than those of the subjects on CPAVI at day $42: 1743.8 \pm 248$ and $1721.3 \pm 250.2$ against $1610.3 \pm 244.9$ g respectively. Apart from the paw yield, the organs were heavier with CPAVI. At the economic level, profits of 484 CFA francs, 345 CFA francs and 281 CFA francs were obtained on treatments SR21, ESPOIR and CPAVI respectively. ESPOIR and SR21 corn varieties, rich in protein, offer definite economic benefits for broiler production. Therefore, the two varieties "SR21" and "ESPOIR" maize are better than maize all coming in the broiler diet.

(C) 2018 International Formulae Group. All rights reserved.

Keywords: Maize, ESPOIR, SR21, proteins, cobb-500, Burkina Faso.

\section{INTRODUCTION}

Situé au cœur de l'Afrique de l'Ouest, le Burkina Faso est un pays à vocation agropastorale. L'agriculture et l'élevage occupent $86 \%$ de la population (CILSS et al., 2006). Le sous-secteur de l'élevage contribue à hauteur de $18 \%$ à la formation de la valeur ajoutée nationale (MRA, 2010). Avec un cheptel national estimé en 2014 par le MRA (2015), à 9091000 bovins, 13891000 caprins, 9278000 ovins, 2346000 porcins, 8468000 pintades et 33752000 poulets. L'aviculture joue un rôle important à la formation de cette richesse nationale de par l'importance de son effectif.

L'aviculture occupe une place importante surtout en milieu rural où les volailles sont élevées non seulement pour la consommation locale, les pratiques rituelles, coutumières, religieuses et sociales, mais aussi pour la génération de revenus à travers la vente (Pousga et Boly, 2009). Cette aviculture est essentiellement traditionnelle et occupe 99,8\% du marché national (MASA, 2013) de volaille mais reste faiblement productive. Face à cette faible productivité, autour des centres urbains se développent l'aviculture moderne Mais cette aviculture se trouve confronté à plusieurs difficultés notamment celle liée à l'alimentation. Au Burkina Faso, cette contrainte alimentaire est plus marquée au niveau des protéines d'origine animale qui apportent les acides aminés indispensables (Hien et al., 2011). C'est pourquoi certains estiment que l'une des solutions résiderait dans l'incorporation d'une ressource végétale locale riche en protéines dans la ration alimentaire des volailles (Nworgu et Fasoghon, 2007 ; Hien et al., 2017). C'est le cas de Boutonnet et al. (2000) qui avaient affirmé qu'un effort de recherche en nutrition nécessite d'être fait pour promouvoir la définition de modèles alimentaires valorisant beaucoup plus les ressources alimentaires ou plantes localement disponibles et bon marché. C'est dans ce contexte que s'inscrit cette présente étude dont l'objectif est de déterminer les effets de deux variétés sélectionnées de maïs à savoir le maïs ESPOIR et SR21 sur la productivité du poulet de chair de souche cobb-500.

\section{MATERIEL ET METHODES \\ Le site d'étude}

Le site du Centre de Promotion de l'Aviculture Villageoise (CPAVI) a accueilli les travaux de l'étude expérimentale. Le CPAVI est un centre qui relève du ministère des ressources animale et halieutique. Ce centre a pour mission d'accompagner les acteurs de la filière et de capitaliser les acquis des différents projets et programmes dans le domaine de l'amélioration de l'aviculture traditionnelle. Le CPAVI se trouve dans l'enceinte de la direction provinciale de l'élevage du Houet. Cette direction se situe au nord de la ville de Bobo-Dioulasso sur la route de Dédougou.

\section{Matériel \\ Poulailler}

Un poulailler de $10 \mathrm{~m}$ sur $5 \mathrm{~m}$ a été aménagé en 09 boxes de $4 \mathrm{~m}^{2}$ chacun. Les 
boxes sont faits en grillage avec une hauteur de $1,5 \mathrm{~m}$ et chaque boxe contenait 23 poulets. La ventilation du bâtiment est naturelle avec une orientation Nord-Sud des façades. La litière faite de balles de riz recouvrait le sol et était renouvelée toutes les deux semaines.

\section{Matériel animal}

Deux cent sept (207) poussins de souche cobb-500 ont été utilisés.

\begin{abstract}
Aliment
L'unité de fabrique d'aliment du Centre de la Promotion de l'Aviculture Villageoise (CPAVI) a fourni les aliments utilisés au cours de l'expérience. Les différentes rations y ont été formulées à partir des variétés de maïs «ESPOIR » et « SR21 » et du maïs « CPAVI» qui est le maïs tout venant ordinairement utilisé par cette unité. Le Tableau 1 présente leur composition chimique. Une même formule alimentaire a été utilisée dans les rations constituant les différents traitements. Les ingrédients utilisés sont : le maïs, le tourteau de soja, la farine d'arachide, le son de blé, la méthionine, le phosphate bi-calcique, les coquilles d'huitre, le sel $(\mathrm{NaCl})$, le prémix chair. Les valeurs bromatologiques de ces différentes rations sont dans le tableau 2.
\end{abstract}

\section{Matériel d'élevage}

Une mangeoire et un abreuvoir d'une capacité de 10 litres ont été disposés dans chaque lot

\section{Produits vétérinaires et sanitaires}

La désinfection a été faite avec l'eau de javel et le virunet pendant toute la durée de l'expérience. Des anti-stress (complexes vitaminés) et des antibiotiques (oxytétracycline, sulfamide) ont été utilisés. Les vaccins contre la maladie de Newcastle et celle de Gumboro ont été également utilisés.

\section{Matériel de pesées}

Le poids des aliments, le poids des médicaments, le poids vifs des poulets, ainsi que le poids de la carcasse ont été prises avec une balance électronique de précision $0,1 \mathrm{~g}$.

\section{Fiches de suivi}

Il s'agit des fiches de consommation d'aliment, de prise de poids, de fiches de mortalité et d'abattage.

\section{Méthodes}

\section{Constitution des lots}

Deux cent sept (207) poussins d'un jour de poids moyen $41,8 \mathrm{~g}$, de souche Cobb500 ont été répartis de façon aléatoire en 9 lots de 23 poulets chacun, puis 3 lots ont aussi été assignés de façon aléatoire à recevoir un des traitements ESPOIR, SR21 et CPAVI (Figure $1)$.

\section{Distribution de l'aliment et de l'eau}

L'eau et l'aliment étaient distribués le matin et à volonté (ad libitum) à $08 \mathrm{H} 00$ et les refus d'aliment étaient pesés afin de déterminer la quantité d'aliment ingérée.

\section{Pesées des animaux}

Les animaux ont été pesés le $1^{\text {er }}$, le $14^{\text {ème }}$, le $29^{\text {ème }}$ jour et le $42^{\text {ème }}$ jour. Le $14^{\text {ème }}$ jour correspondant à la fin de la phase démarrage, le $29^{\text {ème }}$ jour, à celle de la croissance et le $42^{\text {ème }}$ jour, à celle de la finition. Les pesés se faisait le matin avant la distribution de l'aliment.

Le $50^{\text {ème }}$ jour, 10 poulets par lot ont été aléatoirement prélevés et abattus et le poids vif, le poids carcasse, le poids des intestins vides, du gésier vide, des pattes, du foie et de la tête ont été enregistrés.

\section{Paramètres calculés}

L'ingestion d'aliment : la quantité d'aliment ingérée (QAI) a été calculée à partir de la formule suivante:

$Q A I=\frac{Q A D-R F}{\text { Effectif }}$

Avec $\mathrm{QAD}=$ quantité d'aliment distribuée quotidiennement et $\mathrm{RF}=$ refus alimentaire.

Le gain moyen quotidien (GMQ) : Il est calculé à partir des poids vif (PV).

$G M Q=\frac{P V j-P V i}{\text { nombre de jours entre les dates i et } j}$

L'indice de consommation (IC): a été calculé à partir de la formule suivante :

$I C=\frac{Q A T(g)}{\text { Gain de poids }(q) \text { sur la même période }}$

Le rendement carcasse (RC) et le rendement des organes (RO) ont été calculés selon la formule suivante : 
$R C=\frac{P C}{P V} * 100$

Avec $\mathrm{PC}=$ Poids Carcasse, $\mathrm{PO}=$ Poids Organe et $\mathrm{PV}=$ Poids Vif

$R O=\frac{P O}{\mathrm{PV}} * 100$

Le taux de mortalité par traitement : a été calculé sur la base des mortalités enregistrées quotidiennement selon la formule :

Taux de mortalité $=\frac{\text { Nombre de morts dans chaque lot }}{\text { Effectif du lot au début de l'expérience }}$

\section{Rentabilité économique}

Les formules suivantes ont été utilisées :

Coût alimentaire $/$ phase $=$ QAIt $*$ prix du $\mathrm{kg}$ d'aliment Avec QAIt = la Quantité Alimentaire Ingérée totale par phase (Démarrage, croissance et finition).

Coût alimentaire $=\sum$ Coût alimentaire/phase
Prix de vente du poulet $=P V *$ prix du $\mathrm{kg}$ à la ferme Vente de poulets $=$ Nombre de poulets $*$ prix de vente du poulet Amortissement $=\frac{\text { Prix d'achat }}{\text { Durée de vie (jour) }} * 42$

La durée de vie du matériel d'élevage (abreuvoir, mangeoire et chaudière) a été estimée à 2 ans.

Coût de production $=\sum$ charges
Résultat $=$ produits - coût de production

\section{Traitement et Analyse de données}

Les données ont été collectées à l'aide des logiciels Excel 2013. Le traitement de donnée a été réalisé à l'aide du logiciel XLSTAT 2007.7. L'analyse de variance a été faite par la méthode ANOVA. La séparation des moyennes a été réalisée grâce au test de Fisher au seuil de $5 \%$.

Tableau 1 : Composition chimique.

\begin{tabular}{llllllll}
\hline \multirow{2}{*}{ Nature } & Variété & \%MS & $\mathbf{\% M M}$ & $\begin{array}{l}\text { Phosphore total } \\
\mathbf{m g} / \mathbf{k g}\end{array}$ & $\begin{array}{l}\text { Potassium total } \\
\mathbf{( m g / k g})\end{array}$ & $\mathbf{\%}$ PB & \% MO \\
\hline Grain & SR21 & 89,39 & 3,03 & 4418 & 8400 & 11,158 & 86,36 \\
Grain & ESPOIR & 89,28 & 2,37 & 3761 & 6397 & 11,367 & 86,91 \\
\hline
\end{tabular}

MS : Matière sèche, MM : Matière Minérale, MO : Matière Organique.

Tableau 2 : Valeurs bromatologiques calculées.

\begin{tabular}{|c|c|c|c|c|c|c|c|c|c|}
\hline \multirow{2}{*}{$\begin{array}{l}\text { Valeurs } \\
\text { bromatologiques }\end{array}$} & \multicolumn{3}{|c|}{ Démarrage } & \multicolumn{3}{|c|}{ Croissance } & \multicolumn{3}{|l|}{ Finition } \\
\hline & ESPOIR & CPAVI & SR21 & ESPOIR & CPAVI & SR21 & ESPOIR & CPAVI & SR21 \\
\hline $\mathrm{EM}(\mathrm{kcal} / \mathrm{kg})$ & 3021,73 & 3021,73 & 3021,73 & 3109,03 & 3109,03 & 3109,03 & 3196,13 & 3196,13 & 3196,13 \\
\hline $\mathrm{PB}(\%)$ & 22,23 & 21,06 & 22,08 & 20,79 & 19,13 & 20,64 & 18,65 & 16,87 & 18,49 \\
\hline $\mathrm{FB}(\%)$ & 3,24 & 3,24 & 3,24 & 3,58 & 3,58 & 3,58 & 3,51 & 3,51 & 3,51 \\
\hline Lysine & 0,67 & 0,67 & 0,67 & 0,76 & 0,76 & 0,76 & 0,67 & 0,67 & 0,67 \\
\hline Méthionine & 0,49 & 0,49 & 0,49 & 0,58 & 0,58 & 0,58 & 0,59 & 0,59 & 0,59 \\
\hline Calcium $(\mathrm{Ca})$ & 1,3 & 1,3 & 1,3 & 1,08 & 1,08 & 1,08 & 0,92 & 0,92 & 0,92 \\
\hline Phosphore (P) & 0,51 & 0,51 & 0,51 & 0,44 & 0,44 & 0,44 & 0,36 & 0,36 & 0,36 \\
\hline $\mathrm{EM} / \mathrm{PB}$ & 135,95 & 143,48 & 136,85 & 149,55 & 162,5 & 150,61 & 171,37721 & 189,41 & 172,83 \\
\hline $\mathrm{Ca} / \mathrm{P}$ & 2,55 & 2,55 & 2,55 & 2,45 & 2,45 & 2,45 & 2,52 & 2,52 & 2,52 \\
\hline
\end{tabular}

EM : Energie Métabolisable, PB : Protéine Brute, FB : Fibre Brute. 


\begin{tabular}{|l|l|l|}
\hline Traitement ESPOIR & Traitement CPAVI & Traitement SR21 \\
\hline \multicolumn{3}{|c|}{ Couloir } \\
\hline Traitement SR21 & Traitement CPAVI & Traitement ESPOIR \\
\hline \multicolumn{2}{|c}{ Couloir } & Traitement CPAVI \\
\hline Traitement ESPOIR & Traitement SR21 \\
\hline
\end{tabular}

Figure 1: Dispositif expérimental.

\section{RESULTATS \\ Quantité alimentaire ingérée}

La Figure 2 montre la quantité alimentaire journalière ingérée par poulet au démarrage ([1; $15[$ jours), en croissance ([15; $30[$ jours) et en finition ([30;42] jours). L'analyse statistique n'a pas montré de différence significative entre les traitements $(\mathrm{P}>0,05)$. Tout le long de l'essai, la consommation journalière par poulet a été plus importante au niveau du traitement ESPOIR, suivie du traitement CPAVI; celle de la ration à base de maïs SR21 a été la plus faible. Pour toute la durée de l'expérience, chaque poulet a consommé en moyenne $95,7 \pm 56,6 ; 88,1 \pm 52,2$ et $85,3 \pm 52,2 \mathrm{~g} /$ jour respectivement pour les traitements ESPOIR, CPAVI et SR21.

\section{Indice de consommation (IC)}

L'IC a été en moyenne de 2,2 $\pm 0,6$ pour le traitement ESPOIR; $2,2 \pm 0,5$ pour CPAVI et $2,1 \pm 0,3$ pour SR21. Le test statistique n'a révélé aucune différence significative entre les différents traitements $(\mathrm{P}>$ $0,05)$. En dehors de la phase de croissance, dans l'ensemble, l'IC du traitement ESPOIR a été le plus élevé. A 42 jours, pour produire $1 \mathrm{~kg}$ de viande, les poulets soumis au traitement ESPOIR ont consommé $0,1 \mathrm{~kg}$ de plus que ceux nourris au SR21 et $0,5 \mathrm{~kg}$ de plus que ceux alimentés au CPAVI (Figure 3).

\section{Evolution des poids vifs moyens (PVM)}

L'évolution des PVM poulets est présentée dans le Tableau 3. A l'installation, les poussins avaient un poids homogène $(p>0,05)$. Dans l'ensemble, la vitesse de croissance a été faible du $1^{\mathrm{er}}$ au $14^{\text {ème }}$ jour à partir duquel, elle a connu une plus grande augmentation jusqu'au $42^{\text {ème }}$ jour. La courbe du traitement ESPOIR est restée au-dessus des deux autres tout le long de l'essai. A 42 jours, le traitement SR21 semble rattraper le traitement ESPOIR.

A la fin de la période de démarrage, les poulets pesaient en moyenne $266,4 \mathrm{~g}$. Les poulets sous traitement ESPOIR pesaient plus que ceux sous SR21 $(\mathrm{p}<0,05)$. Le traitement CPAVI a obtenu un poids intermédiaire. Les poulets sous traitement ESPOIR ont pesé $28,3 \mathrm{~g}$ de plus que ceux nourris au maïs SR21 et $6,1 \mathrm{~g}$ de plus que le témoin (CPAVI).

A la fin de la phase croissance, le PVM de ESPOIR a été supérieur $(\mathrm{p}<0,05)$ aux deux autres qui ont été statistiquement similaires entre eux.

A la fin de l'expérience (42 jours), le poids des poulets nourris au maïs SR21 a presque rattrapé celui des poulets alimentés au maïs ESPOIR. Les PVM des deux traitements ont été significativement supérieur à celui du CPAVI. Cette différence se traduit au $42^{\text {ème }}$ jour par un gain de 133,5 g des poulets nourris au maiis ESPOIR sur ceux ayant subi le traitement CPAVI.

\section{Gain moyen quotidien (GMQ)}

Le GMQ moyen des animaux pour tout l'essai, était de 40,5 $\pm 19,8 \mathrm{~g} /$ jour pour le traitement ESPOIR ; 40,9 $\pm 22,8$ pour SR21 et de 38,3 \pm 19,6 g/jour pour le témoin (CPAVI). Statistiquement, les traitements n'ont pas eu d'effets significatifs sur le GMQ des oiseaux (P $>0,05)$. Cependant, le maïs ESPOIR a induit la plus grande vitesse de croissance au démarrage et en croissance (Figure 4). En finition, le traitement SR21 a plutôt donné le meilleur GMQ ; $61,3 \pm 21,7$ contre $55,3 \pm 18,7$ et 
$55,5 \pm 19,3 \mathrm{~g} /$ jour pour les traitements ESPOIR et CPAVI respectivement.

\section{Rendement carcasse et rendement en organes}

Les différents traitements n'ont pas eu d'effets significatifs sur le rendement carcasse et sur le pourcentage des pattes $(\mathrm{P}>0,05)$. Les pourcentages du cœur, du gésier vide, de l'intestin vide, du foie et de la tete par rapport au poids vif des poulets nourris avec la ration CPAVI ont été statistiquement supérieurs à ceux des poulets alimentés avec les deux autres rations (Tableau 4). Statistiquement, les rations ESPOIR et SR21 ont eu les mêmes effets sur le rendement carcasse et sur les pourcentages des organes par rapport au poids vif.

\section{Taux de mortalité}

Le taux de mortalité global pour l'ensemble des traitements durant les 42 jours de l'essai a été de $6,3 \%$. Le taux de mortalité le plus élevé a été enregistré au niveau du traitement ESPOIR (8,7 \%) (Figure 5). Le taux de mortalité le plus faible a été enregistré au niveau du traitement SR21.

\section{Bilan économique}

Le Tableau 5 présente les quantités et les coûts utilisés dans la réalisation du compte d'exploitation. Le prix du $\mathrm{kg}$ de chaque ration a été obtenu par calcul sur la base du prix du $\mathrm{kg}$ des différents ingrédients. Le prix de ces ingrédients a été obtenu lors d'une l'enquête auprès des magasins de vente.

Le Tableau 6 présente le compte d'exploitation au terme de l'expérience. Sur la base du prix de vente à $1500 \mathrm{f} \mathrm{CFA} \mathrm{le} \mathrm{kg} \mathrm{de} \mathrm{PV}$ obtenu au $42^{\text {ème }}$ jour, le traitement SR21 a été le plus rentable. Il a permis d'obtenir comme bénéfice par poulet, $203 \mathrm{f}$ CFA de plus que le traitement témoin et $139 \mathrm{f} \mathrm{CFA}$ de plus que le traitement ESPOIR. Le traitement ESPOIR a quant à lui permis d'obtenir $64 \mathrm{f} \mathrm{CFA} \mathrm{de} \mathrm{plus}$ que le traitement témoin. Le coût de production par poulet a été de $2291 \mathrm{f} \mathrm{CFA,} 2154$ f CFA et de $2118 \mathrm{f}$ CFA pour les traitements ESPOIR, CPAVI et SR21 respectivement.

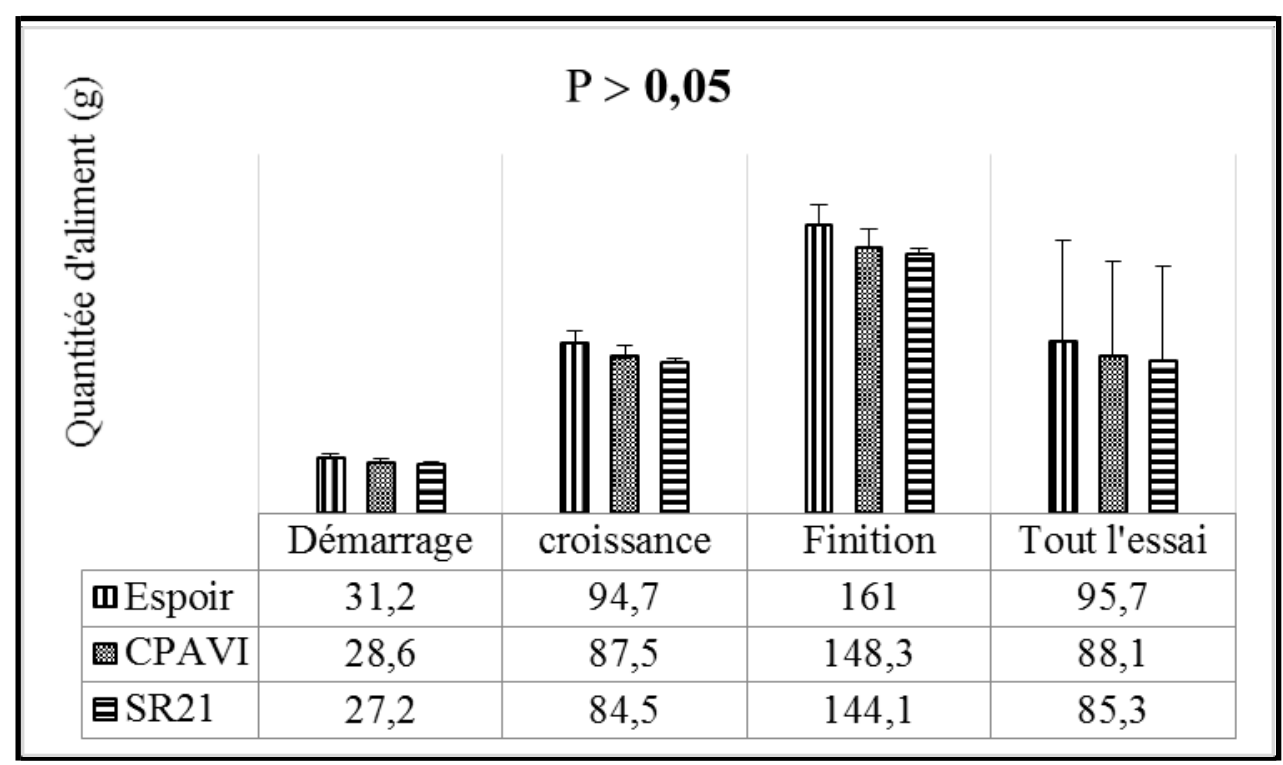

Figure 2: Quantité d'aliment ingérée par poulet par jour. 


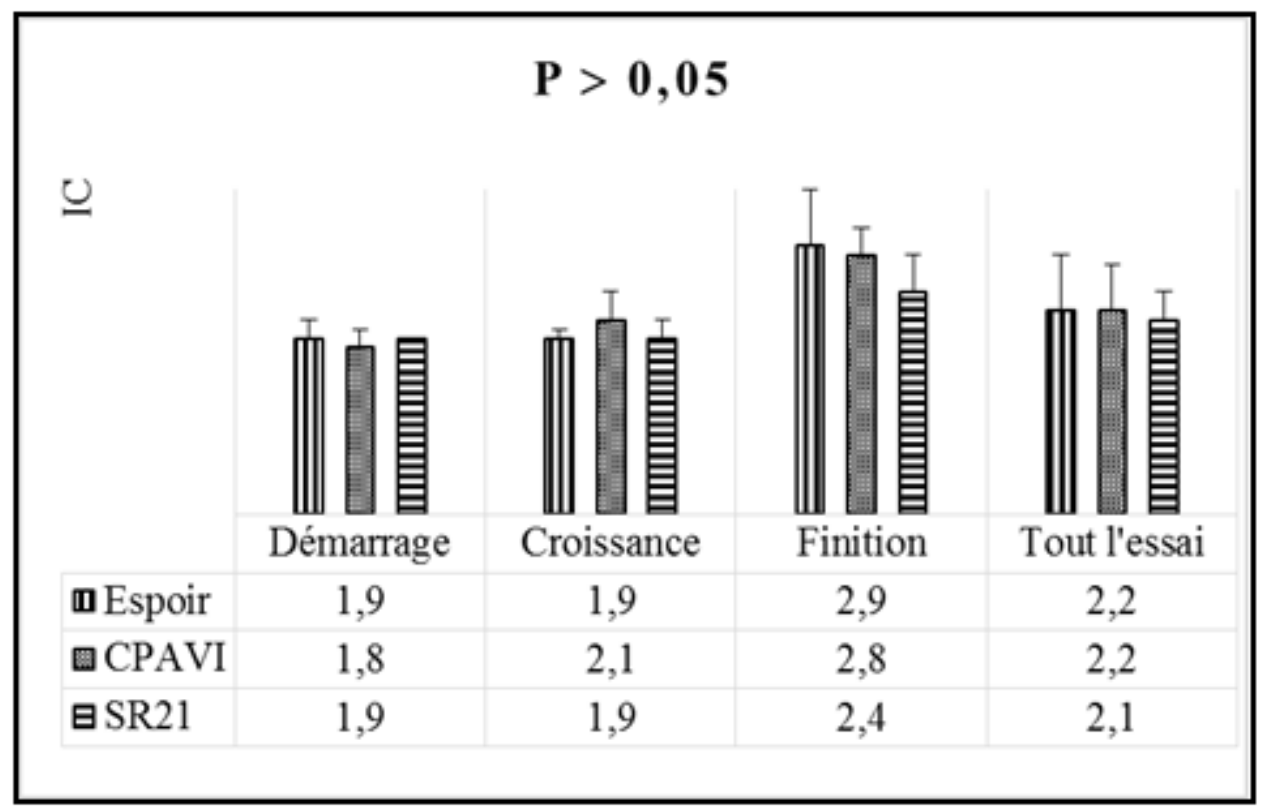

Figure 3: Evolution des indices de consommation.

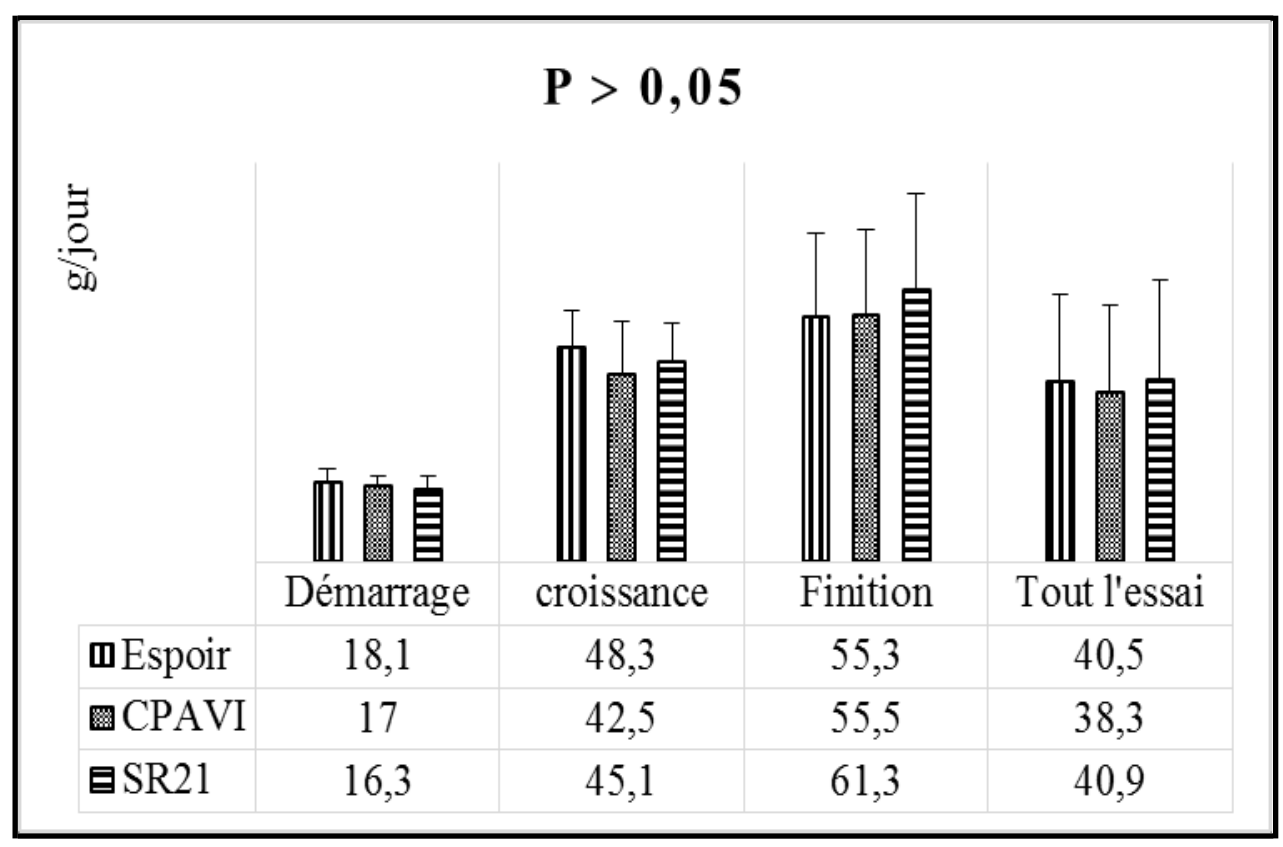

Figure 4: Evolution du gain moyen quotidien. 
Tableau 3 : Poids vif moyen.

\begin{tabular}{lllll}
\hline Traitements & J1 & Démarrage & Croissance & Finition \\
\hline ESPOIR & $41,7 \pm 2,1 \mathrm{a}$ & $272,8 \pm 39,4 \mathrm{a}$ & $999,8 \pm 121,9 \mathrm{a}$ & $1743,8 \pm .248,0 \mathrm{a}$ \\
CPAVI & $42,0 \pm 2,3 \mathrm{a}$ & $266,7 \pm 37,3 \mathrm{ab}$ & $904,9 \pm 127,4 \mathrm{~b}$ & $1610,3 \pm 244,9 \mathrm{~b}$ \\
SR21 & $41,7 \pm 2,3 \mathrm{a}$ & $244,5 \pm 37,2 \mathrm{~b}$ & $931,4 \pm 110,5 \mathrm{~b}$ & $1721,3 \pm 250,2 \mathrm{a}$ \\
\hline
\end{tabular}

Moyenne \pm Ecart-type ;

Sur la même colonne, les valeurs affectées d'une lettre différente sont statistiquement différentes $(p>0,05)$.

Tableau 4: Rendement carcasse et rendement en organes (\%).

\begin{tabular}{lllll}
\hline Paramètres & \multicolumn{2}{l}{ Traitements } & & \multirow{2}{*}{ Pr $>$ F } \\
\cline { 2 - 4 } & ESPOIR & CPAVI & SR21 & \\
\hline Rendement carcasse (\%) & $77,73 \pm 1,94 \mathrm{a}$ & $77,65 \pm 3,46 \mathrm{a}$ & $77,67 \pm 3,51 \mathrm{a}$ & 0,995 \\
\% Cœur & $0,49 \pm 0,11 \mathrm{~b}$ & $0,57 \pm 0,12 \mathrm{a}$ & $0,50 \pm 0,09 \mathrm{~b}$ & 0,007 \\
\% Gésier vide & $2,36 \pm 0,33 \mathrm{~b}$ & $2,57 \pm 0,49 \mathrm{a}$ & $2,36 \pm 0,39 \mathrm{~b}$ & 0,067 \\
\% Intestins vides & $3,59 \pm 0,67 \mathrm{~b}$ & $4,22 \pm 0,64 \mathrm{a}$ & $3,74 \pm 0,63 \mathrm{~b}$ & 0,001 \\
\% Foie & $3,12 \pm 0,54 \mathrm{ab}$ & $3,27 \pm 0,58 \mathrm{a}$ & $2,85 \pm 0,54 \mathrm{~b}$ & 0,015 \\
\% Pattes & $3,85 \pm 0,56 \mathrm{a}$ & $3,98 \pm 0,73 \mathrm{a}$ & $4,02 \pm 0,47 \mathrm{a}$ & 0,551 \\
\% Tête & $2,61 \pm 0,27 \mathrm{~b}$ & $2,95 \pm 0,40 \mathrm{a}$ & $2,63 \pm 0,26 \mathrm{~b}$ & $<0,0001$ \\
\hline
\end{tabular}

Sur la même ligne, les valeurs (moyenne \pm Ecart-type) suivies d'une lettre différente sont significativement différentes $(P<0,05)$.

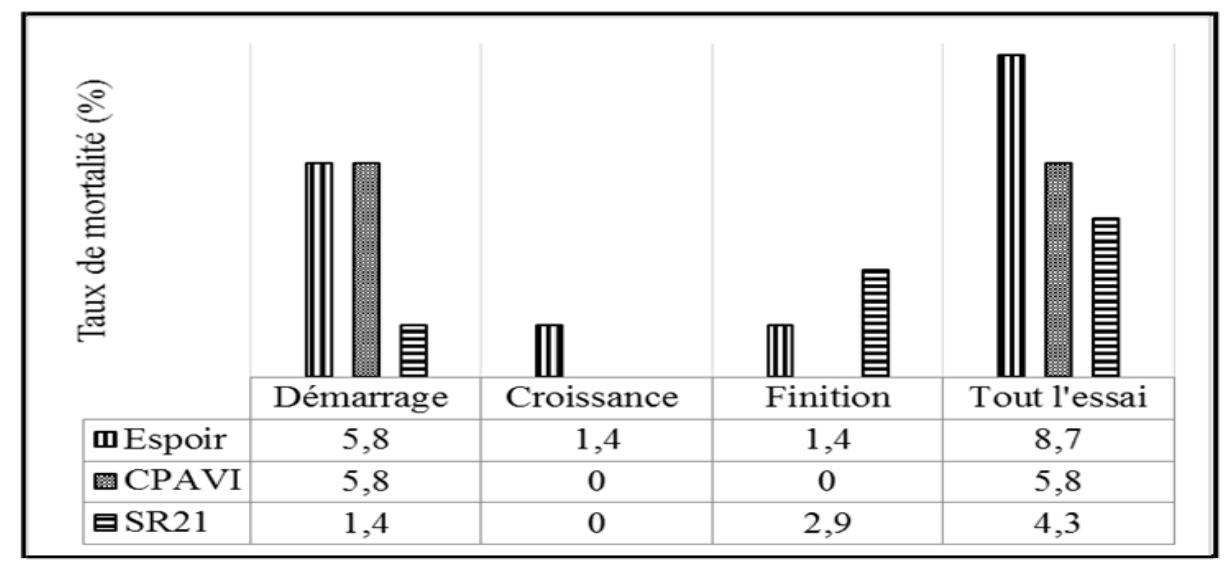

Figure 5 : Taux de mortalité. 
Tableau 5 : Quantités et coûts utilisés dans la réalisation du compte d'exploitation.

\begin{tabular}{lllll}
\hline Désignation & $\begin{array}{l}\text { Quantités } \\
\text { ESPOIR }\end{array}$ & CPAVI & SR21 & $\begin{array}{l}\text { Prix unitaire } \\
\text { (f CFA) }\end{array}$ \\
\hline Poussins achetés & 69 & 69 & 69 & 700 \\
Poulets vendus & 63 & 65 & 66 & $1500 / \mathrm{kg} \mathrm{de} \mathrm{PV}$ \\
Achat de litière (sac de 100 kg) & 1 & 1 & 1 & $300 / \mathrm{sac}$ \\
Vente de litière (sac de 100 kg) & 1,3 & 1,3 & 1,3 & $1000 / \mathrm{sac}$ \\
Aliment démarrage $(\mathrm{kg})$ & 28,4 & 26 & 25,9 & $326 / \mathrm{kg}$ \\
Aliment croissance $(\mathrm{kg})$ & 90,9 & 85,3 & 86,2 & $303 / \mathrm{kg}$ \\
Aliment finition $(\mathrm{kg})$ & 131,9 & 125,3 & 123,6 & $286 / \mathrm{kg}$ \\
\hline
\end{tabular}

Tableau 6 : Compte d'exploitation.

\begin{tabular}{|c|c|c|c|c|c|c|}
\hline \multirow{2}{*}{ Désignation } & \multicolumn{3}{|c|}{ Charges en f CFA } & \multicolumn{3}{|c|}{ Produits en f CFA } \\
\hline & Espoir & CPAVI & SR21 & Espoir & CPAVI & SR21 \\
\hline Achat poussins & 48300 & 48300 & 48300 & & & \\
\hline Vente poulets & & & & 164808 & 156975 & 170412 \\
\hline Aliment & 74514 & 70174 & 69918 & & & \\
\hline Produits vétérinaires & 6667 & 6667 & 6667 & & & \\
\hline Litière & 200 & 200 & 200 & 1300 & 1300 & 1300 \\
\hline Charbon & 1167 & 1167 & 1167 & & & \\
\hline Amortissement bâtiment & $(\mathrm{PM})$ & $(\mathrm{PM})$ & $(\mathrm{PM})$ & & & \\
\hline Amortissement matériel & 182 & 182 & 182 & & & \\
\hline Main d'œuvre & 13333 & 13333 & 13333 & & & \\
\hline Résultat & 21745 & 18252 & 31945 & & & \\
\hline Total & 166108 & 158275 & 171712 & 166108 & 158275 & 171712 \\
\hline
\end{tabular}




\section{DISCUSSION}

\section{Quantité d'aliment ingérée}

Les traitements n'ont pas eu d'effets significatifs sur l'ingestion alimentaire $(\mathrm{P}$ $0,05)$. Tout le long de l'essai, chaque poulet a consommé par jour en moyenne $95,7 \mathrm{~g}$ pour le traitement ESPOIR ; 88,1 g pour le traitement CPAVI et $85,30 \mathrm{~g}$ pour SR21. Ces résultats sont inférieurs à ceux de Sanni (2014) qui avait trouvé une consommation alimentaire journalière par poulet variant de 98,73 à 100,45 g. Cela pourrait s'expliquer par la période de l'expérimentation dont le début a coïncidé avec la fin de la saison hivernale (octobre); période chaude et humide. En effet, en saison chaude (au-delà de $30^{\circ} \mathrm{C}$ ), les besoins énergétiques des poulets diminuent de même que la consommation (Betene A Dooko, 2006); la chaleur réduit les performances du poulet et même augmente son coût de production (Betene A Dooko, 2006).

Même si le test statistique n'a pas montré de différence significative entre les différents traitements, l'aliment à base du maïs jaune (ESPOIR) a été le plus consommé, corroborant les travaux de Hien et al. (2017). Au cours de nos enquêtes il est ressorti que le maïs blanc se pulvérise lorsqu'on l'écrase, ce qui expliquerait que l'aliment à base de maïs blanc SR21 soit moins consommé. Cela pourrait également être expliqué par la coloration jaune de l'aliment ESPOIR qui attirerait les poulets.

En finition (30-42 jours) la consommation moyenne a été de 161 $\mathrm{g} /$ jour/poulet pour ESPOIR, 148,3 g pour CPAVI et $144,1 \mathrm{~g}$ pour SR21. Cette moyenne est supérieure au $125 \mathrm{~g} /$ jour/poulet de Dayon et Arbelot (1997) et au $105 \mathrm{~g}$ enregistrés par Houndonougbo et al. (2012) sur une pondeuse adulte ; elle est légèrement supérieure à celle rapportée par le Mémento de l'agronome (2002) (130 à $150 \mathrm{~g})$.

\section{Indice de consommation}

Les IC ont été Statistiquement identiques pour tous les traitements. L'IC moyen des différents traitements se situe entre 2,1 et 2,2. Selon Abdelouahab (2008), l'IC mesure la rentabilité économique de l'aliment et serait lié à son niveau d'énergie; il mentionne que le développement corporel du poulet de chair est d'autant plus rapide que la consommation quotidienne d'énergie métabolisable est élevée. Ainsi, Ciewe Ciake (2006), après avoir relevé le niveau d'énergie de l'aliment avec de l'huile végétale, a obtenu des IC plus faibles que les nôtres $(1,86$ à 1,96 contre 2,1 à 2,2 ). Nos résultats sont également supérieurs à ceux de Ntivuguruzwa (2008) qui a trouvé des IC de 1,9 à 2. Mais ils sont similaires à ceux de Ngueba Mombo (2006) et inférieurs à ceux de Sanni (2014) (2,5 à 2,72). Le niveau d'énergie des rations utilisées est donc dans la fourchette acceptable en zone tropicale. La différence d'ingestion s'est répercutée sur l'IC. Ainsi sur toute la durée de l'essai, le traitement SR21 a permis d'obtenir le meilleur IC. Autrement, les poulets nourris avec la ration SR21 ont produit plus de viande en consommant moins d'aliment. Le maïs SR 21 (grain blanc) serait mieux valorisé par les poulets de chair que le maïs ESPOIR (grain jaune) et le témoin (grain tout venant).

\section{Evolution des poids vifs moyens}

Le poids vif moyen à l'installation a été de $41,7 \mathrm{~g}$ pour les traitements ESPOIR et SR21 et de 42,0 $\mathrm{g}$ pour CPAVI. Ces poids sont bien compris dans l'intervalle (38-45) indiqué par le Mémento de l'agronome (2002) pour les climats chauds. A 14 jours les poulets nourris, au maïs ESPOIR ont obtenu un poids plus élevé. Les besoins en protéines du poulet de chair sont plus élevés en phase de démarrage, 22\% selon le Mémento de l'Agronome (2002). Le maïs ESPOIR légèrement plus riche en protéines que le maiis SR21 pourrait expliquer cette différence. Le traitement ESPOIR a gardé cet avantage jusqu'à la fin de l'expérience même s'il semble être rattrapé par le traitement SR21 au 42 ${ }^{\text {ème }}$ jour.

Les PVM obtenus (1610,3 à 1743,8 g) sont conformes aux indications du Mémento de l'Agronome (2002) (1600 à $1900 \mathrm{~g}$ ) pour les pays chauds. Ils sont aussi similaires à ceux de Ngueba Mombo (2006) (1660 à 1740 g). Mais 
sont supérieurs à ceux de Betene A Dooko (2006) qui avait trouvé des performances de $1111,47 \mathrm{~g}$ en saison chaude et $1159,02 \mathrm{~g}$ en saison froide. Ces résultats restent tout de même inférieurs à ceux de Ciewe Ciake (2006) $(2120,15$ - 2280,42 g), Ntivuguruzwa (2008) (2405,2 - 2501,9 g), Hien et al. (2010-2012) (2242 à $2328 \mathrm{~g})$ et Sanni (2014) (2085,79 à 2126,06 g). Des PVM plus élevés ont été obtenus alors que la même souche (cobb-500) et le même site ont été utilisés (Hien et al., 2010-2012). Cela pourrait s'expliquer par la faible ingestion alimentaire et par le nonrespect de la prophylaxie sanitaire. En effet, avant l'essai, le poulailler a connu plusieurs bandes des poulets de chair sans observation de vide sanitaire. Cela a sans doute favorisé la prolifération des agents pathogènes réduisant ainsi les performances des animaux.

\section{Gain moyen quotidien}

Les variétés de maïs n'ont pas eu d'effets significatifs sur la vitesse de croissance des poulets de chair $(\mathrm{P}>0,05)$. Les GMQ moyens des animaux étaient de 40,5 g/jour pour le traitement ESPOIR, de 38,3 pour CPAVI 40,9 pour SR21. Les GMQ obtenus sont inférieurs à ceux de Sanni (2014) (48,06 à 49,60 g/jour). L'utilisation de litière de meilleure qualité microbiologique par Sanni pour mener ses expériences, pourrait expliquer cette différence. Ces résultats sont nettement inférieurs à ceux de Ntivuguruzwa (2008) qui avait utilisé des substances à effets anaboliques telles que l'infusé des racines entières de Nauclea latifolia et l'énanthate de testostérone. Ces substances lui ont permis d'obtenir des GMQ de 81,56 à 85,17 g/jour. Nos résultats sont, par contre, comparables à ceux de Hien et al. (2011) dont les travaux sur la valeur nutritive du maïs et du sorgho dans l'alimentation du poulet de chair ont permis d'obtenir en moyenne 54 g/jour à 42 jours d'âge. En phase finition, le maïs SR21 a induit la meilleure vitesse de croissance alors que la consommation alimentaire quotidienne par poulet y était la moins élevée $(144,1$ $\mathrm{g} /$ jour/poulet contre 148,3 et $161 \mathrm{~g} / \mathrm{jour} /$ poulet pour les rations CPAVI et ESPOIR respectivement). Le maïs SR21 serait donc mieux valorisé par les poulets de chair en phase de finition.

\section{Carcasse}

Les variétés de maïs n'ont pas eu d'effet sur le rendement carcasse et sur le poids relatif des pattes. Par contre, la proportion des organes par rapport au poids vif, des poulets nourris avec le maïs ordinaire (CPAVI) a été plus élevée que celle des organes des poulets alimentés avec les rations à base des deux variétés sélectionnées de mais (ESPOIR et SR21). Les rendements carcasses ont été de $77,73 \%$ pour la ration ESPOIR, 77,65\% pour CPAVI et $77,67 \%$ pour SR21. Notre résultat est supérieur à celui de (Hien et al., 2010-012) qui a trouvé des rendements carcasses de 75,25 à $77,14 \%$. Cela pourrait s'expliquer par la durée d'élevage qui a été de 50 jours contre 42 jours pour Hien et collaborateurs. Ce résultat est également supérieur à celui de Kana et al. (2015) qui, après avoir substitué le maïs par la patate à des taux variant de 0 à $100 \%$, avaient trouvé des rendements carcasses de 68,11 à $73,13 \%$. Ces auteurs avaient conclu qu'au-delà de $50 \%$, la patate diminue le rendement carcasse des poulets. Selon ces mêmes auteurs, cette baisse serait liée à la faible digestibilité des glucides contenus dans la patate. La faible proportion du poids du gésier et des intestins des poulets nourris à base de maïs ESPOIR et SR21 montre que ces deux variétés sont plus assimilables par les poulets de chair. En effet, selon Kana et al. (2015), le poids relatif des organes de la digestion augmente avec la teneur en matière indigestible de l'aliment. Ce résultat corrobore ceux de Traoré (2011), selon lesquels le maïs ESPOIR est plus assimilable par l'homme et par les animaux.

\section{Taux de mortalité}

Au cours de l'expérience, le taux de mortalité global était de $6,28 \%$. Nous n'avons pris en compte que les mortalités survenues entre le $1^{\text {er }}$ et le $42^{\mathrm{e}}$ jour. Ce taux est dans la fourchette (5 à $8 \%$ ) acceptable (Mémento de l'Agronome, 2002). Ce résultat reste tout de même inférieur au 10,2\% relevé par Zongo 
(2016) mais supérieur au 1,2\% de Hien et al. (2011) et aux 3,5\% de Ntivuguruzwa (2008). L'augmentation du taux de mortalité global par rapport à celui de Hien et al. (2010-2012) pourra s'expliquer par le fait que le poulailler n'a pas connu de vide sanitaire depuis plusieurs années et par conséquent, était exposé à une probable multiplication des germes pathogènes. En effet, à la $7^{\text {ème }}$ semaine, les poulets ont connu une attaque de la maladie de Newcastle bien que la prophylaxie ait été suivie à la lettre.

\section{Bilan économique}

Les deux variétés de maïs (ESPOIR et SR21), fruits d'une amélioration végétale ont permis d'obtenir le meilleur profit par rapport au maïs ordinaire (tout venant) vendu sur le marché. Cependant, malgré l'obtention d'un poids vif plus élevé, le maïs ESPOIR n'a pas été plus rentable que le maïs SR21. Ces résultats sont à relativiser car nos calculs n'ont pas pris en compte les réalités du terrain. En effet, les poulets ont été vendus à raison de 1500 francs CFA le kg de poids vif. L'enquête a, par contre, montré que plus de la moitié $(52,9 \%)$ des producteurs vendent le poulet par tête. Dans ce cas, seules les conduites qui permettent d'avoir le nombre de poulets le plus élevé en finition sont les plus rentables. De plus, nous avons utilisé deux variétés de maïs sélectionnées pour leur teneur élevée en protéines. Sur le plan économique, ces deux variétés de maïs ont été plus rentables que le maïs vendu sur le marché.

\section{Conclusion}

L'objectif de l'étude était d'appréhender l'effet des variétés de maïs ESPOIR et SR21 sur les performances zootechniques du poulet de chair de souche cobb-500. Les résultats montrent que les variétés de maïs ESPOIR et SR21 ont eu des effets significativement similaires sur les performances zootechniques du poulet de chair. La consommation alimentaire individuelle par jour, l'IC, le GMQ et le rendement carcasse ont été statistiquement similaires pour tous les traitements au seuil de
5\%. Les maïs ESPOIR et SR21 ont donné des PVM à 42 jours statistiquement similaires de $1743,8 \pm 248 \mathrm{~g}$ et $1721,3 \pm 250,2 \mathrm{~g}$ respectivement. Mais ces PVM ont été supérieurs à celui du témoin $(1610,3 \pm 244,9$ g). Par contre en dehors du rendement des pattes, c'est le traitement CPAVI qui a donné les rendements en organes les plus élevés. Sur le plan économique, le maïs SR21 a été le plus rentable. Des bénéfices de 484 , de 345 et de $281 \mathrm{f}$ CFA ont été obtenus pour le traitement SR21, ESPOIR et CPAVI respectivement. Par conséquent, pour augmenter la rentabilité économique des fermes avicoles, ces variétés de maïs riches en protéines (ESPOIR et SR21) doivent être utilisées dans les rations alimentaires. Ce travail mérite d'être poursuivi par l'étude d'autres variétés de maïs sélectionnées afin d'identifier celles qui pourraient permettre aux poulets d'optimiser au plan nutritionnel et économique.

\section{CONFLIT D'INTERETS}

Les auteurs déclarent qu'il n'y a aucun conflit d'intérêts pour cet article.

\section{CONTRIBUTIONS DES AUTERURS}

Cette étude a été financée par West African Sorghum research Network (WCARSN) à travers Dr HANCOCK J.D. et Dr SALISSOU ISSA. Elle a été conduite par OUATTARA Lamine et OUEDRAOGO Arouna Sous l'encadrement de Dr HIEN Ollo Chérubin et Dr DIARRA Boureima.

\section{REMERCIMENTS}

Les auteurs adressent leurs sincères remercîments à West African Sorghum research Network (WCARSN) à travers Dr HANCOCK J.D. Departement of Animal Science and Industry, Manhatan pour l'intérêt qu'il porte à la recherche en en Afrique. 


\section{REFERENCES}

Abdelouahab O. 2008. Le soja dans l'alimentation du poulet de chair aspects qualitatif et quantitatif, Université Mentouri De Constantine Faculté Des Sciences (Département Des Sciences Vétérinaires El-Khroub), mémoire de magister en médecine vétérinaire, Algérie, 72p.

Betene A, Dooko CL. 2006. Evaluation des performances zootechniques et économiques en période post reforme d'élevage de poulets de chair (souches cobb-500 et jupiter) dans la région de Dakar. Thèse de Médecine Vétérinaire, EISMV/UCADD, Dakar ; Sénégal, 115p.

Boutonnet JP, griffon M, Viallet D. 2000. Compétitive des productions animales en Afrique subsaharienne et à Madagascar, Direction Générale de la Coopération internationale et du Développement, 103.

Ciewe Ciake S. 2006. Evaluation de l'effet de la nature et du niveau de la matière grasse alimentaire sur la productivité du poulet de chair. Thèse de médecine vétérinaire, EISMV/UCADD, Dakar ; Sénégal, 100p.

CILSS, CEDEAO, MRA, MAHRH, FEWS NET/USAID, 2006. Impacts socioéconomiques de la grippe aviaire en Afrique de l'Ouest: «Etude de cas au Burkina Faso» Draft. Burkina Faso. 69 p.

Hien OC, Diarra B, Coulibay Y, 2017. Effets d'une ration à base de la variété de maïs « Espoir » sur la productivité des poulettes Int. J. Biol. Chem. Sci., 11(2): $\quad 806-816 . \quad$ DOI: https://dx.doi.org/10.4314/ijbcs.v11i2.2 2

Hien OC, Salissou I, Diarra B, Sanon pp, Hancock JD, 2010 - 2012. Etude comparée de la valeur nutritive du maïs et du sorgho blanc dans l'alimentation des poulets de chair. Science et Technique, Sciences Naturelles et Agronomie, 32(1-2) : 7-20.

Hien OC, Bougouma VMC, Somé W. 2011.
Effets de la substitution de la farine de poisson par celle de chenilles de karité (Cirina butyrospermivuillet) sur la croissance et les paramètres de ponte chez des poules de souche Isa-brown. Science et Technique, Sciences Naturelles et Agronomie, 32(1-2).

Houndonougbo MF, Chrysostome CAAM, Houndonougbo VP. 2012. Performances bioéconomiques des poulettes alimentées avec des rations à base de feuilles séchées de manioc (Manhiot esculenta). Int. J. Biol. Chem. Sci., $\quad$ 6(2): 670-676. http://ajol.info/index.php/ijbcs.

Kana JR, Doue M, Kreman K, Diarra M, Mube K H, Ngouana T, Teguia A. 2015. Effet du taux d'incorporation de la farine de patate douce crue dans l'aliment sur les performances de croissance du poulet de chair. Journal of Applied Biosciences, 91: 8539 - 8546.

MASA. 2013. Situation de référence des principales filières animales au Burkina Faso. MASA, Burkina Faso, 162p.

Mémento de l'Agronome, 2002. Cirad / Ministère Français des Affaires Etrangères, Edition du GRET, Paris. $1692 \mathrm{p}$.

MRA. 2010. Politique nationale de développement durable de l'élevage au Burkina Faso 2010-2025. MRA, Ouagadougou, Burkina Faso, 54p.

MRA. 2015. Annuaires des statistiques de l'élevage 2014. MRA, Ouagadougou, Burkina Faso, 177 p.

Ngueba Mombo L. 2006. L'influence de la substitution du mais par le niébé sur les performances de croissance du poulet de chair en milieu tropical sec. Thèse de médecine vétérinaire, EISMV/UCADD, Dakar, Sénégal, 86p.

Ntivuguruzwa JB. 2008. Effets de l'infuse des racines entieres de Nauclea latifolia (sm) sur les performances de croissance du poulet de chair. Thèse de médecine vétérinaire, EISMV/UCADD, Dakar ; Sénégal, $103 p$.

Nworgu FC, Fasoghon FO. 2007. 
Centrosema (Centrosema pubescens) leaf meal as protein suplement for pullet chicks and growing pullets. Int. J. Biol. Chem. Sci., 6(4): 255-260.

Pousga S, Boly H. 2009. Synthèse des travaux de recherche en aviculture au Burkina Faso ; Rapport de recherche $\mathrm{N}^{\circ} 4$. In Avicuture familiale, 18(1\&2), FAO ; 28-34.

Sanni JY. 2014. Effets d'une litière à base d'attapulgite calcinée, sur les performances de croissance du poulet de chair. Thèse de doctorat en médicine vétérinaire, EISMV/UCADD, Dakar, Sénégal, 88p.
Traoré BA. 2011. Amélioration de la variété de maïs (Zea mays L). ESPOIR en vue de l'intensification de la maïsiculture au Burkina Faso. Identification des composantes essentielles. Mémoire du Diplôme d'Ingénieur du Développement Rural, IDR/UPB ; BoboDioulasso, Burkina Faso ,74 p

Zongo R. 2016. Etude comparée de la rentabilité économique des poulets de chair alimentés avec des rations comprenant des ingrédients industriels. Mémoire du Diplôme d'Ingénieur du Développement Rural, IDR/UPB, Bobo- Dioulasso, Burkina Faso, 85p. 